\title{
The learning curve as a measure of experience
}

\author{
Bertrand D Guillonneau
}

In surgical training, learning curves are used to plot the number of attempts required to master a difficult procedure, or the number of procedures required to achieve proficiency. It is incorrect to describe a procedure that is difficult to learn as having a 'steep' learning curve, as this implies that large gains in proficiency are achieved over a small number of cases. Instead, the curve for a procedure that requires a lot of cases to reach proficiency should be described as 'flattened'. Learning curves evaluate one parameter at a time and not a procedure in its entirety. They are personal, a graph of an individual surgeon's progress. Before the introduction of learning curves, mastery of a surgical technique was judged by one's teachers and peers, and included the accumulation of knowledge, apprenticeship with an experienced surgeon and practice under supervision.

It would be convenient if we could assert that after a given number of patients, a surgeon's learning curve would reach the renowned 'plateau' of proficiency. This, however, is not possible or desirable because it depends primarily on the surgeon's own objectives, such as the level of quality they want to achieve. Also, there are several learning curves for a given procedure, each of which progresses at its own rate, sometimes independently of the others. Today, we prefer it if obstacles can be overcome quickly. Is it possible that endurance is no longer a valued quality? In surgery, however, endurance might be exactly what is needed.

Not all interventions can be taught by employing learning curves. They are not suitable for procedures that have to be completely successful from the first attempt, because failure would entail unacceptable complications. Learning curves are widely used in the teaching of radical prostatectomy, perhaps because of the prolonged natural history of prostate tumors.

It is important to ensure that learning curves are not used to justify our need for 'hands on' experience. Focusing too closely on learning curves leads to acceptance and tolerance of
It is the

responsibility

of surgeon-

teachers to

ensure their

teaching is of

a high quality,

and that they

share their

experience

with students, only using

learning curves

in the service

of patients

or else to

eliminate their

use.

BD Guillonneau is

Head of the Section of

Minimally Invasive

Surgery, within

the Department of

Urology at Memorial

Sloan-Kettering

Cancer Center, New

York.

\section{Competing interests}

The author declared he has

no competing interests.

www.nature.com/clinicalpractice doi:10.1038/ncpuro0249 unexpected negative results under the false notion that these are necessary during the process of acquiring certain skills; this should not be tolerated by urologists or patients. This is where experience matters and prevails, as it can be shared and taught.

Teaching should be centered on techniques and results. How can one teach without the false security of learning curves, whilst ensuring that urologists will know when they have reached the best standard of surgery that patients have a right to expect? There are probably several answers, but surely an education based exclusively on technique can only lead to an impasse. The patient and his or her disease must remain the basis for teaching, even when the surgeon is super-specialized. Some think that the introduction of technology will allow superspecialization to be dispensed with. This view is mistaken, as the problem is not the means by which a procedure is conducted, but the results attained. Waiting for new technologies is misleading to patients and surgeons. It is uncertain whether developments such as minifellowships, observerships, surgery schools, and other 'hands-on' sessions are the best way of providing quality teaching. Are they not, in fact, only ways of discarding the responsibility of teachers, deferring them on to students?

It is the responsibility of surgeon-teachers to ensure their teaching is of a high quality, and that they share their experience with students, only using learning curves in the service of patients or else to eliminate their use. The model of the learning curve is outdated and no longer acceptable. Learning should involve spending time with someone who has experience and accepts the responsibility of sharing it. This is the eternal concept of teaching, its privilege, honor and duty.

In the recurring debates comparing the outdated use of learning curves to measure mastery versus experience, experience will prevail because it is indispensable to both surgeons and patients. 